\title{
Simple trace criterion for classification of multilayers
}

\author{
L. L. Sánchez-Soto, J. J. Monzón, T. Yonte \\ Departamento de Óptica, Facultad de Ciencias Físicas, \\ Universidad Complutense, 28040 Madrid, Spain * \\ J. F. Cariñena \\ Departamento de Física Teórica, Facultad de Ciencias, \\ Universidad de Zaragoza, 50009 Zaragoza, Spain
}

(Dated: October 26, 2018)

\begin{abstract}
The action of any lossless multilayer is described by a transfer matrix that can be factorized in terms of three basic matrices. We introduce a simple trace criterion that classifies multilayers in three classes with properties closely related with one (and only one) of these three basic matrices.

PACS numbers:
\end{abstract}

Any linear system with two input and two output channels can be described in terms of a $2 \times 2$ transfer matrix [1, 2]. In many instances (e.g., in polarization optics [3]), we are interested in the transformation properties of quotients of variables rather than on the variables themselves. Then, the transfer matrix induces a bilinear transformation that represents the action of the system in the complex plane [4].

For layered media the use of $2 \times 2$ matrix methods is standard, but the corresponding complex-plane representation is not a common tool. In fact, it has been recently established that for a lossless multilayer the transfer matrix is an element of the group $\mathrm{SU}(1,1)[5]$, which is the key for a deeper understanding of its behavior and simplifies exceedingly its description in terms of bilinear transformations.

Moreover, as we have pointed out recently [6], the Iwasawa decomposition provides a remarkable factorization of the transfer matrix representing any multilayer (no matter how complicated it could be) as the product of three matrices of simple interpretation. At the geometrical level, such a decomposition translates directly into three actions that are the basic bricks from which any multilayer action is built.

In this Letter we go one step further and show that the trace of the transfer matrix allows for a classification of multilayers in three disjoint classes with properties very close to those appearing in the Iwasawa decomposition.

To maintain the discussion as self contained as possible we briefly summarize the essential ingredients of multilayer optics we shall need for our purposes. The configuration we consider is a stratified structure that consists of a stack of plane-parallel layers sandwiched between two semi-infinite ambient $(a)$ and substrate $(s)$ media, which we shall assume to be identical, since this is the common experimental case. Hereafter all the media are supposed to be lossless, homogeneous, and isotropic.

We assume an incident monochromatic, linearly polarized plane wave from the ambient, which makes an angle $\theta_{0}$ with the normal to the first interface and has ampli- tude $E_{a}^{(+)}$. The electric field is either in the plane of incidence ( $p$ polarization) or perpendicular to the plane of incidence ( $s$ polarization). We consider as well another plane wave of the same frequency and polarization, and with amplitude $E_{s}^{(-)}$, incident from the substrate at the same angle $\theta_{0}$ [f].

As a result of multiple reflections in all the interfaces, we have a backward-traveling plane wave in the ambient, denoted $E_{a}^{(-)}$, and a forward-traveling plane wave in the substrate, denoted $E_{s}^{(+)}$. It is useful to treat the field amplitudes as the vector

$$
\mathbf{E}=\left(\begin{array}{c}
E^{(+)} \\
E^{(-)}
\end{array}\right)
$$

which applies to both ambient and substrate media. Then, the amplitudes of the fields at each side of the multilayer are related by a $2 \times 2$ complex matrix $\mathrm{M}_{a s}$, we shall call a multilayer transfer matrix [8], in the form

$$
\mathbf{E}_{a}=\mathrm{M}_{a s} \mathbf{E}_{s}
$$

where, in our case, $\mathrm{M}_{a s}$ can be expressed as

$$
\mathrm{M}_{a s}=\left[\begin{array}{cc}
1 / T_{a s} & R_{a s}^{*} / T_{a s}^{*} \\
R_{a s} / T_{a s} & 1 / T_{a s}^{*}
\end{array}\right] \equiv\left[\begin{array}{cc}
\alpha & \beta \\
\beta^{*} & \alpha^{*}
\end{array}\right]
$$

with $\operatorname{det} \mathrm{M}_{a s}=+1$, which shows that the set of lossless multilayer matrices reduces to the group $\mathrm{SU}(1,1)$, whose elements depend on three independent real parameters. In this matrix $R_{a s}$ and $T_{a s}$ are, respectively, the overall reflection and transmission coefficients for a wave incident from the ambient.

For the case at hand, the Iwasawa decomposition reads as 6

$$
\mathrm{M}_{a s}=\mathrm{K}(\phi) \mathrm{A}(\xi) \mathrm{N}(\nu)
$$

where

$$
\mathrm{K}(\phi)=\left[\begin{array}{cc}
\exp (i \phi / 2) & 0 \\
0 & \exp (-i \phi / 2)
\end{array}\right]
$$




$$
\begin{aligned}
& \mathrm{A}(\xi)=\left[\begin{array}{cc}
\cosh (\xi / 2) & i \sinh (\xi / 2) \\
-i \sinh (\xi / 2) & \cosh (\xi / 2)
\end{array}\right], \\
& \mathrm{N}(\nu)=\left[\begin{array}{cc}
1-i \nu / 2 & \nu / 2 \\
\nu / 2 & 1+i \nu / 2
\end{array}\right] .
\end{aligned}
$$

In other words, $\mathrm{K}(\phi)$ belongs to a compact, $\mathrm{A}(\xi)$ to an abelian, and $\mathrm{N}(\nu)$ to a nilpotent subgroup, respectively, and their physical meaning has been discussed in Ref. [6]. Furthermore, such a decomposition is global and unique 9].

As we have said, we are often interested in the transformation properties of field quotients rather than the fields themselves. Therefore, it seems natural to consider the complex numbers

$$
z=\frac{E^{(-)}}{E^{(+)}}
$$

for both ambient and substrate. From a geometrical viewpoint, Eq. (2) defines a transformation of the complex plane $\mathbb{C}$, mapping the point $z_{s}$ into the point $z_{a}$, according to

$$
z_{a}=\Phi\left[\mathrm{M}_{a s}, z_{s}\right]=\frac{\beta^{*}+\alpha^{*} z_{s}}{\alpha+\beta z_{s}} .
$$

Thus, the action of the multilayer can be seen as a function $z_{a}=f\left(z_{s}\right)$ that can be appropriately called the multilayer transfer function [3, 10]. The action of the inverse matrix $\mathrm{M}_{a s}^{-1}$ is $z_{s}=\Phi\left[\mathrm{M}_{a s}^{-1}, z_{a}\right]$.

We can define an action of the group $\mathrm{SU}(1,1)$ on the complex plane $\mathbb{C}$ by these bilinear transformations. The complex plane appears then decomposed in three regions that remain invariant under the action of the group: the unit disc, its boundary and the external region [10].

The Iwasawa decomposition has an immediate translation in this geometrical framework, and one is led to treat separately the action of each one of the matrices appearing in this decomposition. To this end, it is worth noting that the group $\mathrm{SU}(1,1)$ we are considering appears always as a group of transformations of the complex plane. The concept of orbit is especially appropriate for obtaining an intuitive picture of the corresponding action. We recall that, given a point $z$, its orbit is the set of points $z^{\prime}$ obtained from $z$ by the action of all the elements of the group. In Fig. 1 we have plotted some orbits for each one of the subgroups of matrices $\mathrm{K}(\phi), \mathrm{A}(\xi)$, and $\mathrm{N}(\nu)$. For matrices $\mathrm{K}(\phi)$ the orbits are circumferences centered at the origin, for $\mathrm{A}(\xi)$, they are arcs of circumference going from the point $+i$ to the point $-i$ through $z$. Finally, for the matrices $\mathrm{N}(\nu)$ the orbits are circumferences passing through the point $+i$ and joining the points $z$ and $-z^{*}$.

To go beyond this geometrical picture of multilayers, let us introduce the following classification: a matrix is of class $K$ when $\left[\operatorname{Tr}\left(\mathrm{M}_{a s}\right)\right]^{2}<4$, is of class $A$ when $\left[\operatorname{Tr}\left(\mathrm{M}_{a s}\right)\right]^{2}>4$, and finally is of class $N$ when $\left[\operatorname{Tr}\left(\mathrm{M}_{a s}\right)\right]^{2}=4$. To gain insight into this classification, let us also introduce the fixed points [11] of a transfer matrix as the points in the complex plane that are invariant under the action of $\mathrm{M}_{a s}$; i.e.,

$$
z=\Phi\left[\mathrm{M}_{a s}, z\right]
$$

whose solutions are given by

$$
z=\frac{-i \operatorname{Im} \alpha \pm \sqrt{(\operatorname{Re} \alpha)^{2}-1}}{\beta},
$$

Since $\operatorname{Tr}\left(\mathrm{M}_{a s}\right)=2 \operatorname{Re} \alpha$, one easily check that the matrices of class $K$ have two fixed points, one inside and other outside the unit disc, both related by an inversion; the matrices of class $A$ have two fixed points both on the boundary of the unit disc and, finally, the matrices class $N$ have only one (double) fixed point on the boundary of the unit disc.

Now the origin of the notation for the classes should be clear: if one consider the Iwasawa decomposition (何), one can see that the matrices $\mathrm{K}(\phi)$ are of the class $K$ with the origin as the fixed point in the unit disc, matrices $\mathrm{A}(\xi)$ are of the class $A$ with fixed points $+i$ and $-i$ and matrices $\mathrm{N}(\nu)$ are of the class $N$ with the double fixed point $+i$. Of course, this is in agreement with the orbits in Fig. 1.

To proceed further let us note that by taking the conjugate of $\mathrm{M}_{a s}$ with any matrix $\mathrm{C} \in \mathrm{SU}(1,1)$ we obtain another multilayer matrix; i.e.,

$$
\widehat{\mathrm{M}}_{a s}=\mathrm{CM}_{a s} \mathrm{C}^{-1},
$$

such that $\operatorname{Tr}\left(\widehat{\mathrm{M}}_{a s}\right)=\operatorname{Tr}\left(\mathrm{M}_{a s}\right)$. The fixed points of $\widehat{\mathrm{M}}_{a s}$ are then the image by $\mathrm{C}$ of the fixed points of $\mathbf{M}_{a s}$. For our classification viewpoint it is essential to remark that if a multilayer has a transfer matrix in the class $K, A$, or $N$, one can always find a family of matrices $C$ such that the conjugate through $\mathrm{C}$ is of the form $\mathrm{K}(\phi), \mathrm{A}(\xi)$ or $\mathrm{N}(\nu)$, respectively. The explicit construction of this family of matrices is easy: it suffices to impose that $C$ transforms the fixed points of $\mathrm{M}_{a s}$ into the corresponding fixed points of $\mathrm{K}(\phi), \mathrm{A}(\xi)$, or $\mathrm{N}(\nu)$. For example, if $\mathrm{M}_{a s}$ is in the class $K$ and its fixed point inside the unit disc is $z_{f}$, one should have

$$
\Phi\left[\mathrm{CM}_{a s} \mathrm{C}^{-1}, 0\right]=\Phi\left[\mathrm{CM}_{a s}, z_{f}\right]=\Phi\left[\mathrm{C}, z_{f}\right]=0 .
$$

In order to appreciate the physical meaning of this classification let us take another conjugate of Eq. (10) by the unitary matrix

$$
\mathcal{U}=\frac{1}{\sqrt{2}}\left[\begin{array}{ll}
1 & i \\
i & 1
\end{array}\right] .
$$

In consequence, we can rewrite it alternatively as

$$
\mathcal{E}_{a}=\mathcal{M}_{a s} \mathcal{E}_{s}
$$


where the new field vectors are defined as

$$
\mathcal{E}=\left(\begin{array}{c}
\mathcal{E}^{(+)} \\
\mathcal{E}^{(-)}
\end{array}\right)=\mathcal{U}\left(\begin{array}{c}
\widehat{E}^{(+)} \\
\widehat{E}^{(-)}
\end{array}\right)=\mathcal{U C}\left(\begin{array}{c}
E^{(+)} \\
E^{(-)}
\end{array}\right),
$$

and the transformed multilayer matrix reads as

$$
\mathcal{M}_{a s}=\mathcal{U} \widehat{\mathrm{M}}_{a s} \mathcal{U}^{-1}=\mathcal{U C ~} \mathrm{M}_{a s} \mathrm{C}^{-1} \mathcal{U}^{-1}
$$

One can easily check that $\operatorname{det} \mathcal{M}_{a s}=+1$ and all its elements are real numbers. Therefore, $\mathcal{M}_{\text {as }}$ belongs to the group $\mathrm{SL}(2, \mathbb{R})$ that underlies the structure of the celebrated $A B C D$ law in first-order optics [12, 13, 14.

By transforming by $\mathcal{U}$ the Iwasawa decomposition (4), we get the corresponding one for $\operatorname{SL}(2, \mathbb{R})$, which has been previously worked out 15]:

$$
\mathcal{M}_{a s}=\mathcal{K}(\phi) \mathcal{A}(\xi) \mathcal{N}(\nu)
$$

where

$$
\begin{aligned}
\mathcal{K}(\phi) & =\left[\begin{array}{cc}
\cos (\phi / 2) & \sin (\phi / 2) \\
-\sin (\phi / 2) & \cos (\phi / 2)
\end{array}\right] \\
\mathcal{A}(\xi) & =\left[\begin{array}{cc}
\exp (\xi / 2) & 0 \\
0 & \exp (-\xi / 2)
\end{array}\right], \\
\mathcal{N}(\nu) & =\left[\begin{array}{ll}
1 & 0 \\
\nu & 1
\end{array}\right]
\end{aligned}
$$

The physical action of these matrices is clear. Let us consider by the moment all of them as $A B C D$ matrices in geometrical optics that apply to position $\mathbf{x}$ and momentum $\mathbf{p}$ (direction) coordinates of a ray in a transverse plane. These are the natural phase-space variables of ray optics. Then $\mathcal{K}(\phi)$ would represent a rotation in these variables, $\mathcal{A}(\xi)$ a magnifier that scales $\mathbf{x}$ up to the factor $m=\exp (\xi / 2)$ and $\mathbf{p}$ down by the same factor, and $\mathcal{N}(\nu)$ the action of a lens of power $\nu \sqrt{14}$.

In the multilayer picture, $\mathcal{E}^{(+)}$can be seen as the corresponding $\mathbf{x}$, while $\mathcal{E}^{(-)}$can be seen as the corresponding p. Then, the key result of this Letter is that when the multilayer transfer matrix has $\left[\operatorname{Tr}\left(\mathrm{M}_{a s}\right)\right]^{2}$ lesser, greater or equal to 4 one can find in a direct way a family of matrices that gives a new vector basis such that the action of the multilayer, when viewed in such a basis, is exclusively rotationlike, or magnifierlike, or lenslike.

We expect that the formalism presented here could provide a general tool for analyzing and classifying the multilayer performance in an elegant and concise way.

* Electronic address: sanchez@eucmax.sim.ucm.es

[1] P. Yeh, Optical Waves in Layered Media (Wiley, New York, 1988).

[2] J. Lekner, Theory of Reflection (Dordrecht, Amsterdam, 1987).

[3] R. M. A. Azzam and N. M. Bashara, Ellipsometry and Polarized Light (North-Holland, Amsterdam, 1987).
[4] D. Han, Y. S. Kim, and M. E. Noz, Phys. Lett. A 219, 26 (1996).

[5] J. J. Monzón and L. L. Sánchez-Soto, Opt. Commun. 162, 1 (1999); J. Opt. Soc. Am. A 16, 2013 (1999); ibid 16, 2786 (1999).

[6] J. J. Monzón, T. Yonte, and L. L. Sánchez-Soto, Opt. Lett. 26, 370 (2001).

[7] When ambient $(0)$ and substrate $(m+1)$ media are different, the angles $\theta_{0}$ and $\theta_{m+1}$ are conected by Snell law $n_{0} \sin \theta_{0}=n_{m+1} \sin \theta_{m+1}$, where $n_{j}$ denotes the refractive index of the $j$ th medium.

[8] I. Ohlídal and D. Franta, Ellipsometry of Thin Film Systems, Progress in Optics (Edited by E. Wolf) 41, 181 (North-Holland, Amsterdam, 2000).

[9] S. Helgason, Differential Geometry, Lie Groups and Symmetric Spaces (Academic, New York, 1978).

[10] T. Yonte, J. J. Monzón, L. L. Sánchez-Soto, J. F. Cariñena, and C. López-Lacasta, J. Opt. Soc. Am. A (submitted for publication), Los Alamos e-print archive physics/0104050.

[11] V. Bargmann, Ann. Math. 48, 568 (1947).

[12] H. Bacry and M. Cadilhac, Phys. Rev. A 23, 2533 (1981).

[13] R. Simon, N. Mukunda, and E. C. G. Sudarshan, Opt. Commun. 65, 322 (1988).

[14] R. Simon and N. Mukunda, J. Opt. Soc. Am. A 15, 2146 (1998).

[15] R. Simon, E. C. G. Sudarshan, and N. Mukunda, Phys. Rev. A 29, 3273 (1984). 
FIG. 1: Plot of several orbits in the unit disc of the elements of the Iwasawa decomposition $\mathrm{K}(\phi), \mathrm{A}(\xi)$, and $\mathrm{N}(\nu)$ for the group of multilayer transfer matrices. 

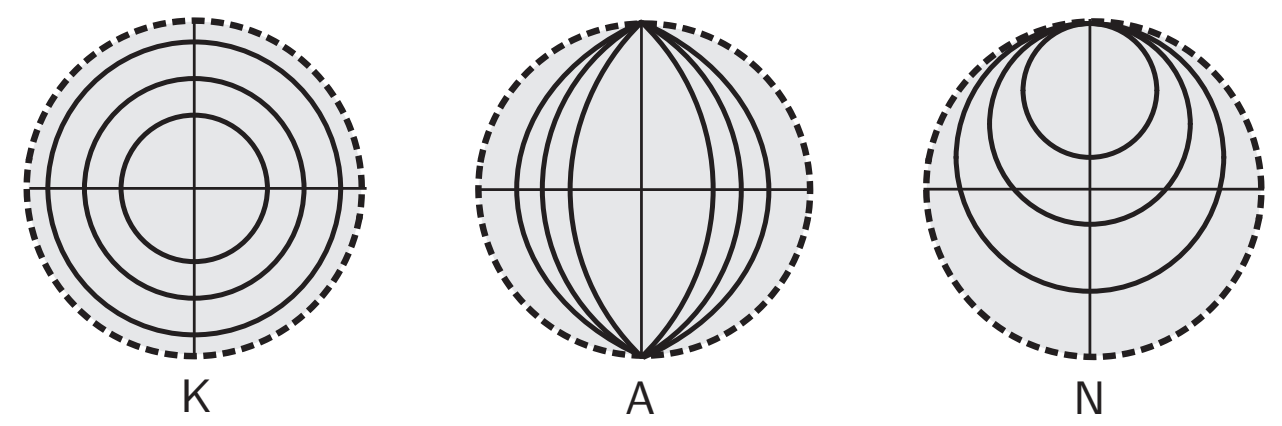